\title{
Hexokinase and phosphofructokinase activity and intracellular distribution correlate with aggressiveness and invasiveness of human breast carcinoma
}

\author{
Raquel G. Coelho ${ }^{1,2,5}$, Isadora C. Calaça ${ }^{1}$, Deborah M. Celestrini' ${ }^{1}$ Ana Helena P. \\ Correia-Carneiro ${ }^{3}$, Mauricio M. Costa ${ }^{3}$, Patricia Zancan ${ }^{4}$, Mauro Sola-Penna ${ }^{1}$ \\ 'Laboratório de Enzimologia e Controle do Metabolismo (LabECoM), Departamento de Biotecnologia Farmacêutica, Faculdade \\ de Farmácia, Universidade Federal do Rio de Janeiro, Rio de Janeiro, Brasil \\ ${ }^{2}$ Instituto de Bioquímica Médica Leopoldo De Meis, Universidade Federal do Rio de Janeiro, Rio de Janeiro, Brasil \\ ${ }^{3}$ Hospital Universitário Clementino Fraga Filho, Universidade Federal do Rio de Janeiro, Rio de Janeiro, Brasil \\ ${ }^{4}$ Laboratório de Oncobiologia Molecular (LabOMol), Departamento de Biotecnologia Farmacêutica, Faculdade de Farmácia, \\ Universidade Federal do Rio de Janeiro, Rio de Janeiro, Brasil \\ ${ }^{5}$ Present address: Laboratório de Fisiologia Endócrina Doris Rosenthal (LFE), Instituto de Biofísica Carlos Chagas Filho, \\ Universidade Federal do Rio de Janeiro, Rio de Janeiro, Brasil
}

Correspondence to:

Mauro Sola-Penna, e-mail: maurosp@ufrj.br

Keywords: glycolysis, prognosis, breast cancer, treatment, diagnosis

Received: May 29, $2015 \quad$ Accepted: July 27, $2015 \quad$ Published: August 07, 2015

\section{ABSTRACT}

Glycolytic enzymes, such as hexokinase and phosphofructokinase, have been reported to be upregulated in many cancer types. Here, we evaluated these two enzymes in $\mathbf{5 4}$ breast cancer samples collected from volunteers subjected to mastectomy, and the results were correlated with the prognosis markers commonly used. We found that both enzymes positively correlate with the major markers for invasiveness and aggressiveness. For invasiveness, the enzymes activities increase in parallel to the tumor size. Moreover, we found augmented activities for both enzymes when the samples were extirpated from patients presenting lymph node involvement or occurrence of metastasis. For aggressiveness, we stained the samples for the estrogen and progesterone receptors, HER-2, p53 and Ki-67. The enzyme activities positively correlated with all markers but Ki-67. Finally, we conclude that these enzymes are good markers for breast cancer prognosis.

\section{INTRODUCTION}

Breast cancer is the most common malignant cancer type in women globally, costing billions of dollars per year for its treatment [1-4]. The success of the treatment is directly correlated to the stage of the cancer upon initial treatment, which depends on how early the cancer has been detected. However, one of the major problems associated with this cancer type is that the strong side effects of the treatment still cause severe discomfort to the patients. [5]. Therefore, breast cancer prevention and treatment programs aim to identify more efficient and precise diagnostic and prognostic markers, as well as targets for a treatment with milder side effects [5].

Approximately $80 \%$ of breast cancers are invasive, breaking though the ductal or glandular walls where they originated. Thus, these two types of invasive breast cancers are designated as Infiltrating Ductal Carcinoma (IDC; $\sim 80 \%$ of invasive breast cancers) and Invasive Lobular Carcinoma (ILC; $\sim 10 \%$ of invasive breast cancers), respectively [4]. According to the stage of invasion, breast cancers are classified as 0 , I, II, III or IV, where 0 is the non-invasive stage and IV is when the cancer has invaded the lymph nodes and other tissues such as the lungs, liver, skin, brain, etc. [6]. In terms of prognosis, breast cancers are classified according to size $(\mathrm{T})$, the involvement of lymph nodes (N) and the occurrence of distant metastasis (M). This TNM classification is widely used, attributing numbers according to the severity of the case [6]; $\mathrm{T}$ is classified from 0-4 where 0 is no evidence of a tumor, $\mathrm{N}$ is classified from $0-3$ and $\mathrm{M}$ is classified as 0 or 1 , 
following the same principle [6, 7]. Additionally, many other markers have been used to determine the prognosis of breast cancer, and among the commonly used markers are whether the tumors are negative or positive for the presence of the following proteins: progesterone and estrogen receptors (PR and ER, respectively), human epidermal growth factor receptor 2 (HER-2), the nuclear protein $\mathrm{Ki}-67$ and the tumor suppressor p53 [8-10]. Normally, tumors containing receptors for the sexual hormones are fast growing, which worsens the prognosis [9]. The same principle is also valid for the presence of HER-2 [9, 11]. However, the presence of these receptors is also used to target drugs directly to tumors. For instance, tamoxifen and trastuzumab are directed toward ER and HER-2 positive cancers, respectively [12-14]. The most aggressive type of breast cancer is negative for PR, ER and HER-2 and is termed the triple negative [9, 11]. In addition, the presence of $\mathrm{Ki}-67$ and/or the absence of p53 are strongly indicative of a highly proliferative and aggressive cancer $[10,15,16]$.

To support the high rate of proliferation, cancers have adapted their metabolism so that they present a unique metabolic profile [16, 17]. Designated the 'Warburg effect', cancers consume large amounts of glucose through glycolysis, mostly producing lactate and even consuming an elevated amount of oxygen [18-24]. Oxygen is consumed due to the high activity of certain reactions in the citric acid cycle that are mainly converting glutamine into carbon skeletons (fatty acids, cholesterol, amino acids, etc.) for biomass production [24]. At the same time, glycolysis furnishes ATP to support metabolism as a whole [19, 22-24]. Many alterations allow these cells to present a high glycolytic profile. Among these alterations, we and other groups have devoted special attention to the intracellular distribution of the key glycolytic enzymes hexokinase (HK) and phosphofructokinase (PFK). Both enzymes present complex regulatory mechanisms, being activated and inhibited by many allosteric ligands. For instance, HK, the first enzyme in the glycolytic pathway, is inhibited by its product, glucose-6-phosphate (G6P). The inhibition of $\mathrm{HK}$ reflects on the decrease of glucose uptake by the cell [25]. Likewise, PFK, the second kinase in glycolysis, is inhibited by one of its substrates, ATP, when at concentrations higher than $1 \mathrm{mM}[26,27]$. Since ATP is also one of the final products of glycolysis, an excess of this metabolite reflects on the inhibition of PFK and the whole glycolysis [28]. On the other hand, these enzymes can associate with other intracellular components, altering their catalytic rates and regulatory properties. For example, the activity of these enzymes is enhanced when HK binds to the voltage-dependent anion channel (VDAC) in mitochondria [25, 29] and when PFK binds to actin filaments (f-actin) in the cytoskeleton [28]. Moreover, these associations reflect on the loss of allosteric inhibition of HK by G6P and of PFK by ATP. It has been shown that the association of HK with VDAC and of PFK with f-actin, namely, particulate HK and PFK, are increased in tumors, compared to non-tumoral tissues $[28,30-40]$. One hypothesis is that increased particulate HK and PFK is part of the mechanism that originates the 'Warburg effect' [41].

In the present study, we aimed to correlate the increased HK and PFK activity with the altered intracellular distribution of the prognosis markers for human breast cancers. We evaluated the enzymes in material (cancerous and non-cancerous tissues) collected from 54 donors subjected to mastectomy after cancer diagnosis. Our results reveal a strong correlation between the particulate fraction of the enzymes and the markers for the most aggressive cancer types.

\section{RESULTS}

The current work has analyzed tumoral and nontumoral (adjacent to tumoral and used as a control) breast tissues from 54 female volunteers (average age $62 \pm 11$ years) subjected to mastectomy at the major hospital of the Federal University of Rio de Janeiro (Hospital Universitário Clementino Fraga Filho - HUCFF/UFRJ) between 2007 and 2009. All the volunteers signed the free and informed consent terms prior to surgery and were aware of all procedures concerning the samples. All samples were morphologically and histologically classified according to the TNM classification, as well as for the presence of ER, PR Her-2, p53 and Ki-67. Additionally, glycolytic markers, including glucose consumption rate, lactate production rate, $\mathrm{HK}$ activity and PFK activity, were measured in tumor cells and compared to the non-tumoral counterparts from the same patient. The results are summarized in Figure 1. Among the tissues collected, $83 \%$ were diagnosed as IDC and $17 \%$ as ILC. IDC samples were further sub-classified as stage I (7\%), II (16\%), III (22\%) and IV (55\%) according to the invasion level of the cancer (Figure 1A). Regarding tumor size, due to the samples characteristics, we subdivided the samples into three groups, where $7 \%$ were less than $2 \mathrm{~cm}$ (T1), $50 \%$ were more than $2 \mathrm{~cm}$ but less than $5 \mathrm{~cm}$ (T2) and $43 \%$ were more than $5 \mathrm{~cm}$ (T3; Figure 1A). Additionally, $46 \%$ of the tumors were triple negative (Figure $1 \mathrm{C}$ ), and $78 \%$ and $46 \%$ of the patients presented compromised axillary lymph nodes and metastasis on skin, respectively (Figure 1D). Furthermore, $70 \%$ and $35 \%$ of the tumors were negative for $\mathrm{p} 53$ and positive ( $>50 \%$ staining) for Ki-67, respectively (Figure 1D). The overall classification analysis revealed that most of the tumors analyzed presented a very aggressive profile. Moreover, compared to the non-tumoral tissues excerpted from the patients, tumors presented elevated glycolytic markers, (Figure $1 \mathrm{E})$ such as glucose consumption rate $(35 \%$ higher; $P<$ $0.05)$, lactate production rate $(3.3$-fold higher; $P<0.05)$, HK activity $(3.5$-fold higher; $P<0.05)$ and PFK activity (2.0-fold higher; $P<0.05$ ). 
A

Tumor classification
B

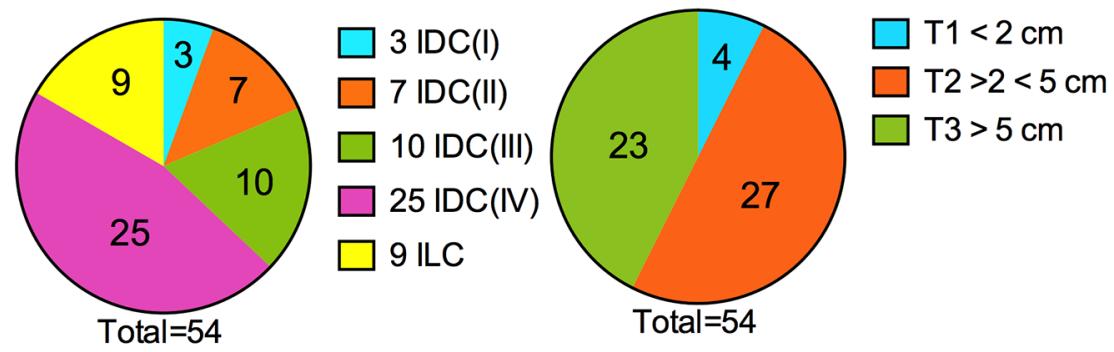

C Hormone receptors

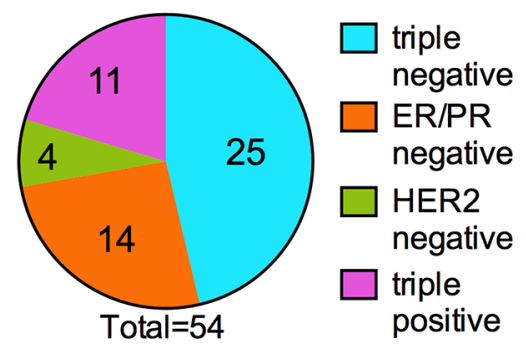

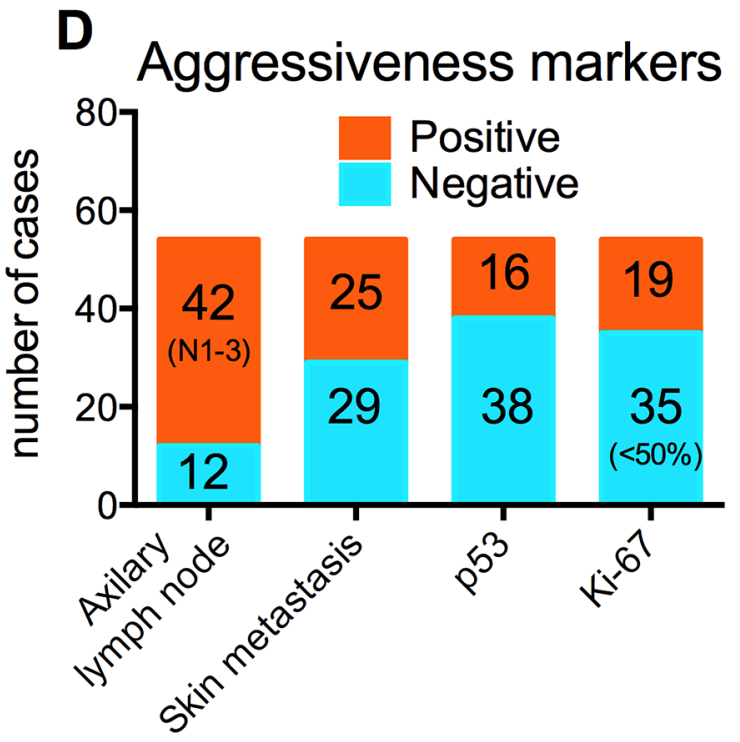

E Glycolytic profile

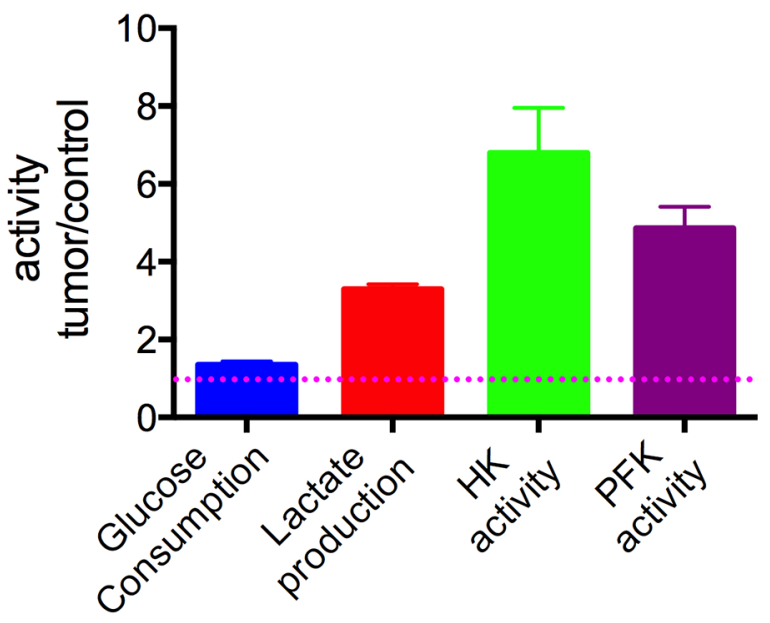

Figure 1: Profile of the breast carcinoma samples. Panel A. Classification of the tumors. Panel B. Size classification according to TNM. Panel C. Responsiveness to the hormone receptors. Panel D. Aggressiveness markers. Panel E. Glycolytic profile. The glycolytic profile was investigated as described in the Materials and Methods. The plotted values are the mean \pm standard errors of at least 40 samples. The dotted line is related to the control values, i.e., when the ratio between the values for non-tumoral tissues and tumoral tissues is equal to 1 . All the plotted values are significantly different from the values obtained with non-tumoral tissues $(P<0.05$, two-way ANOVA, Sidak's post-test).

The activity of HK and PFK was further analyzed in terms of the ratio of the activities of the enzymes in tumor samples relative to non-tumor samples, according to the stage of invasion and TNM classification of the tumors. We analyzed not only the total activity but also the particulate activity of both enzymes. The IDC tumors in stage IV presented much higher HK and PFK activities (total and particulate activities), compared to the others (Figure 2A-2D). Indeed, the enzyme activities among the IDC groups increased proportionally to the invasiveness of the tumors (I $<$ II $<$ III $<$ IV). ILC samples were also analyzed, and they tended to present lower enzyme activities than IDC samples (IV), confirming the proportionality of the invasiveness. There was also a positive correlation between the tumor sizes and the activity of the particulate fraction of both HK and PFK (Figure 3B and 3D, respectively). However, no statistically significant differences were observed in the total activity of the enzymes (Figure 3A and 3C). The activities of both enzymes, either total or particulate, were higher when there is the involvement of lymph nodes (Figure 4) or the occurrence of skin metastasis (Figure 5).

According to the presence of hormone receptors in the tumors, our results clearly show that the triple negative samples present higher activities of HK and PFK (total and particulate), compared to all the other samples (Figure 6A$6 \mathrm{D}$, blue bars). The triple negative was lower than the HER2 positive samples (Figure 6A) only for total HK activity. Indeed, the HER-2 positive tumors presented HK and PFK activities higher than the triple positive and the PR/ ER positive samples, and only the particulate HK activity presented no difference between HER-2 positive and PR/ ER positive samples (Figure 6B). The triple positive and the PR/ER positive samples presented lower activities of 

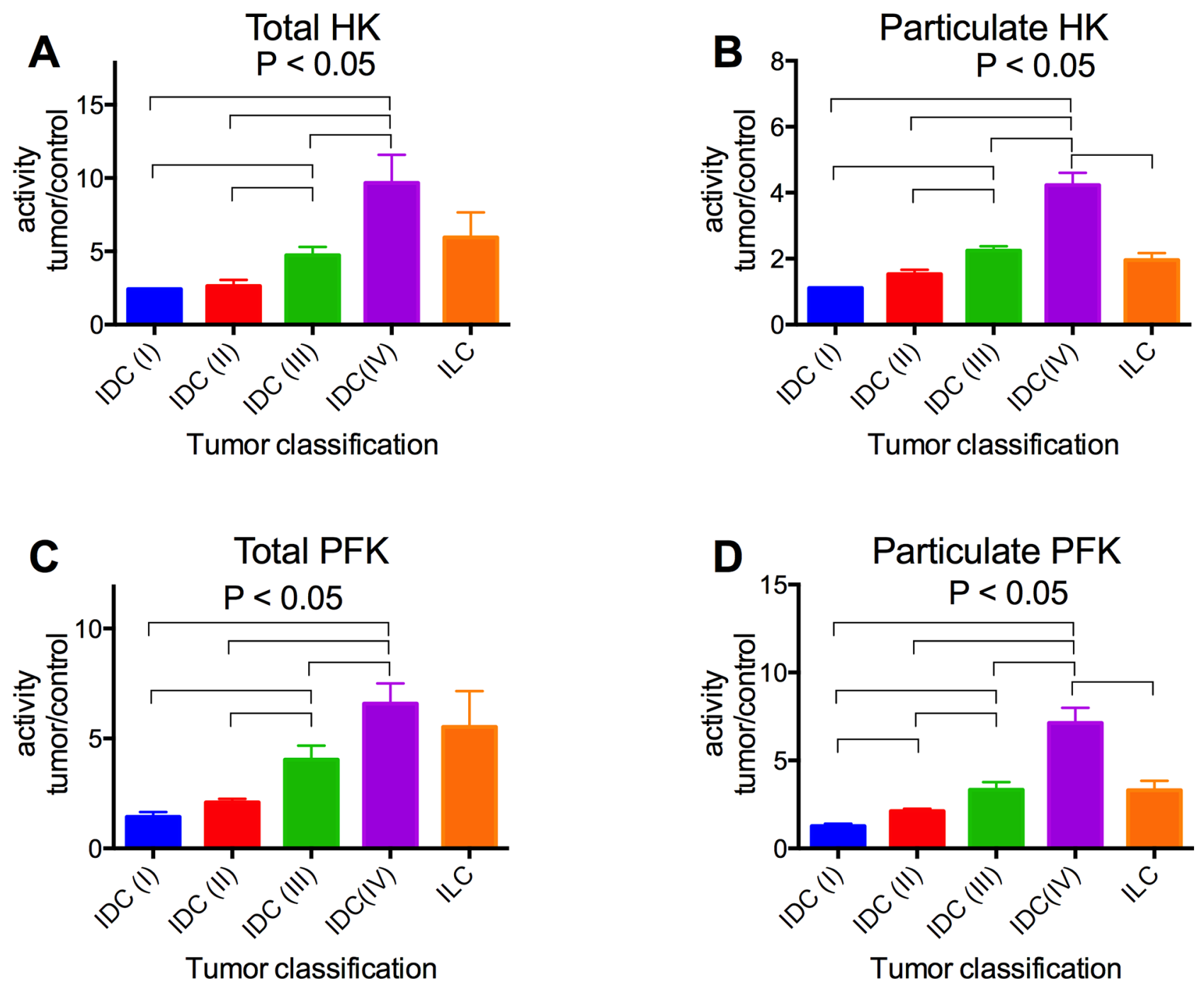

Figure 2: HK and PFK activities of the tumor samples separated with regard to tumor classification. Tumor classification was performed according to invasiveness as described in the Materials and Methods. HK and PFK activity in the total and particulate fractions was analyzed as described in the Materials and Methods. Panel A. Total HK activity. Panel B. Particulate HK activity. Panel C. Total PFK activity. Panel D. Particulate PFK activity. The plotted values are the means \pm standard errors, and the numbers of cases are in accordance with the results presented in Figure 1. Statistical analyses were conducted using ANOVA and Sidak's post-test.

the enzymes and were not different between themselves for all of the analyses (Figure 6A-6D). Comparing the samples that were negative and positive for p53 staining, it was clear that the p53 negative tumors presented higher activities of both enzymes in total and particulate fractions (Figure 7). However, we found no differences in the enzymes comparing Ki-67 negative and positive tumors (Figure 8).

\section{DISCUSSION}

The increased glycolytic profile associated with a high production of lactate, even in the presence of an appropriate supply of oxygen, is one of the hallmarks of cancer [16, 18-20, 22]. This unique altered metabolic profile is the result of several changes and/or mutations in proteins, mainly enzymes, transcription factors/controllers and receptors, that control the metabolic flux [41]. Among these alterations, we have reported that PFK, one of the major regulatory glycolytic enzymes, has altered activity $[31,35]$, expression patterns [32] and intracellular distributions in cancers [30-32, 34, 35]. Moreover, we found that the intracellular distribution alteration, i.e., the association with f-actin within the cytoskeleton, is more pronounced in metastatic cancers compared to nonmetastatic cancers [34, 35]. Although the association between PFK and f-actin is one of the physiological mechanisms of enzyme activation by many stimuli [28, 42-54], the detachment of PFK from f-actin has proven to be lethal for cancer cells but non-lethal to non-tumor cells [31, 34, 36-38, 54]. Following the same pattern, HK associates with mitochondria as an activating mechanism, and this association is more pronounced in cancers [36, $37,40,55]$. The detachment of HK from mitochondria decreases the viability of tumor cells but has no effect on 

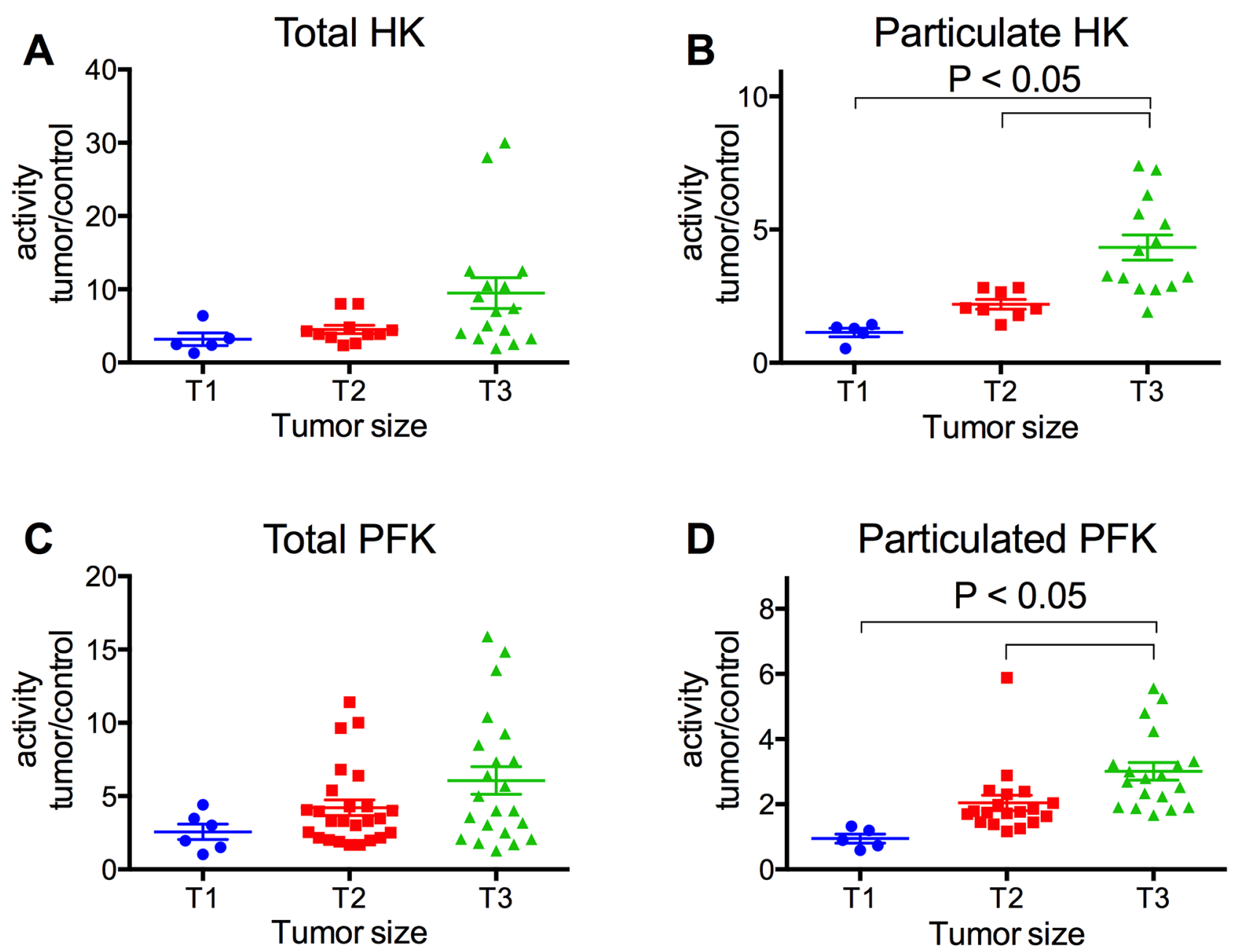

Figure 3: HK and PFK activities of the tumor samples separated with regard to tumor size. Tumor size groups, T1, T2 and T3 were separated as described in the Materials and Methods. HK and PFK activity in the total and particulate fractions was analyzed as described in the Materials and Methods. Panel A. Total HK activity. Panel B. Particulate HK activity. Panel C. Total PFK activity. Panel D. Particulate PFK activity. The plotted values are the mean of enzyme activity evaluated in triplicate for each tumor sample. Statistical analyses were conducted using ANOVA and Sidak's post-test.

non-tumor cells [55-57]. Therefore, it has been previously proposed that the increased activity of HK and PFK, as well as their increased particulate fractions, i.e., f-actinbound PFK or mitochondria-bound $\mathrm{HK}$, is characteristic of tumor cells $[16,18-21,24,32,41,55,57,58]$. Therefore, the current work investigated the correlation between the total activity of these two glycolytic enzymes and their particulate fraction activity, as well as the most commonly used prognostic markers for human breast cancer.

Most of the breast cancer samples we obtained (91\%) were diagnosed as infiltrating ductal carcinoma (IDC), which is compatible with the frequency of this type of cancer $[4,59]$. These samples were further divided by the stage of invasion following the most acceptable guidelines $[7,59]$ among four groups: I, II, III and IV, where I is the least invasive and IV presents metastasis. Our data suggests that HK and PFK activities correlate with the invasiveness of the cancer (Figure 2). The IDC (IV) presented a much higher activity than the other samples, which was also confirmed when the samples were separated as M0 and M1 (non-metastatic or metastatic, respectively; Figure 5). Because tumor invasiveness correlates with tumor size $[2,10]$, it is not surprising that we also found a correlation between enzyme activity and tumor size (Figure 3). In spite of the lack of statistically significant differences among the total enzyme activity when separated by tumor size, the tendency is clearly observed $(P=0.068$ for total HK, Figure $3 \mathrm{~A}$ and 0.057 for total PFK, Figure 3C; two-way ANOVA), and the increase is supported by the particulate enzymes activity results (Figure 3B and 3D). Moreover, an additional marker for breast tumor aggressiveness is the presence of tumor cells within the axillary lymph nodes $[2,10]$. When our samples were classified according to this marker, we observed an increased activity of both HK and PFK, in the total and particulate fractions (Figure 4). Taken together, the results presented in Figures 2, 3, 4 and 5 suggest that HK and PFK activity directly correlates to breast cancer tumor aggressiveness. 

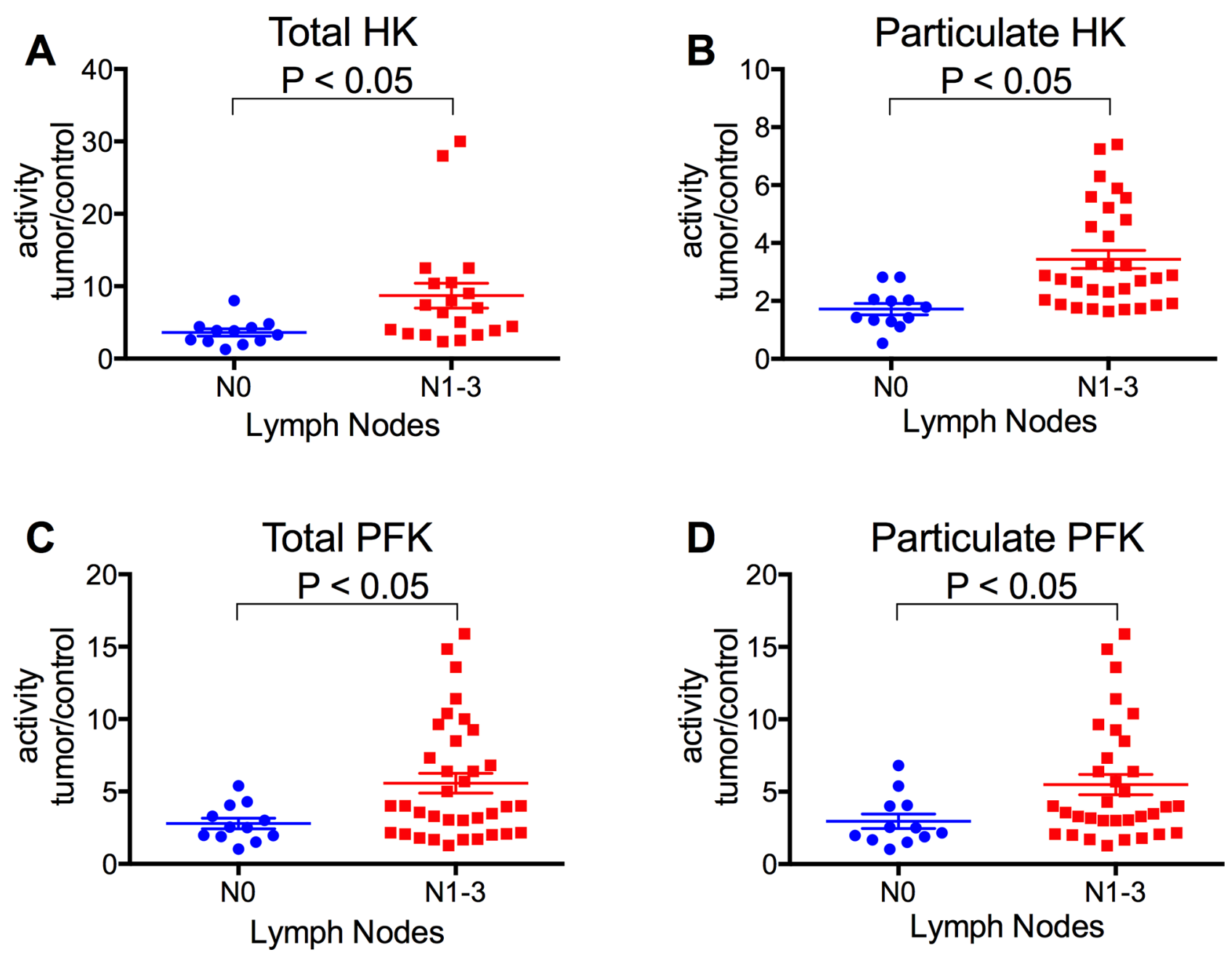

Figure 4: HK and PFK activities of the tumor samples separated with regard to the presence of cancer cells in the lymph nodes. Lymph nodes were analyzed as described in the Materials and Methods. HK and PFK activity in the total and particulate fractions was analyzed as described in the Materials and Methods. Panel A. Total HK activity. Panel B. Particulate HK activity. Panel C. Total PFK activity. Panel D. Particulate PFK activity. The plotted values are the mean of enzyme activity evaluated in triplicate for each tumor sample. Statistical analyses were conducted using ANOVA and Sidak's post-test.

Different biochemical markers such as ER, PR, HER-2, p53 and Ki-67 have been used to determine the prognosis of breast cancers [2]. However, the conclusion about the relevance and significance of the results of these markers is still unclear [60]. However, it is clear that the triple negative breast cancer lacking ER, PR and HER-2 is the cancer presenting the worst prognosis, and usually presents a more aggressive behavior and poorer outcome when compared to the others [61]. The triple negative samples from the current work also presented the higher enzyme activities, either in total and particulate fractions, compared to the ER/PR positive, HER-2 positive or the triple positive samples (Figure 6). Only for the total HK activity is the triple negative not significantly different from the HER-2 positive (Figure 6A, $P=0.079$, two-way ANOVA, Sidak's post-test). Although not significant, the results suggest a tendency because the activity for the triple negative and HER-2 positive samples is different for the HK particulate fraction (Figure 6B). Moreover, the statistical analysis was potentially compromised because we only analyzed 4 HER-2 positive samples. However, the fact that the HER-2 positive samples, which are considered to present a worse prognosis than the ER/PR positive and triple positive tumors, presented the second highest HK and PFK activity strengthens the correlation between the enzyme activity and the prognosis for breast cancer patients. The same conclusion can be reached when the samples are separated into p53 negative and positive, which present worse and better prognoses, respectively [62]. With regard to HK and PFK activity, p53 negative samples presented the higher activity (Figure 7), resulting in, again, the worse outcome. However, we found no correlation between HK and PFK activities and Ki-67 expression in our samples (Figure 8). Although recommended as a prognostic marker, the relevance and significance of this marker is still under scrutiny, and this marker has been used only in association with other markers [63, 64]. 

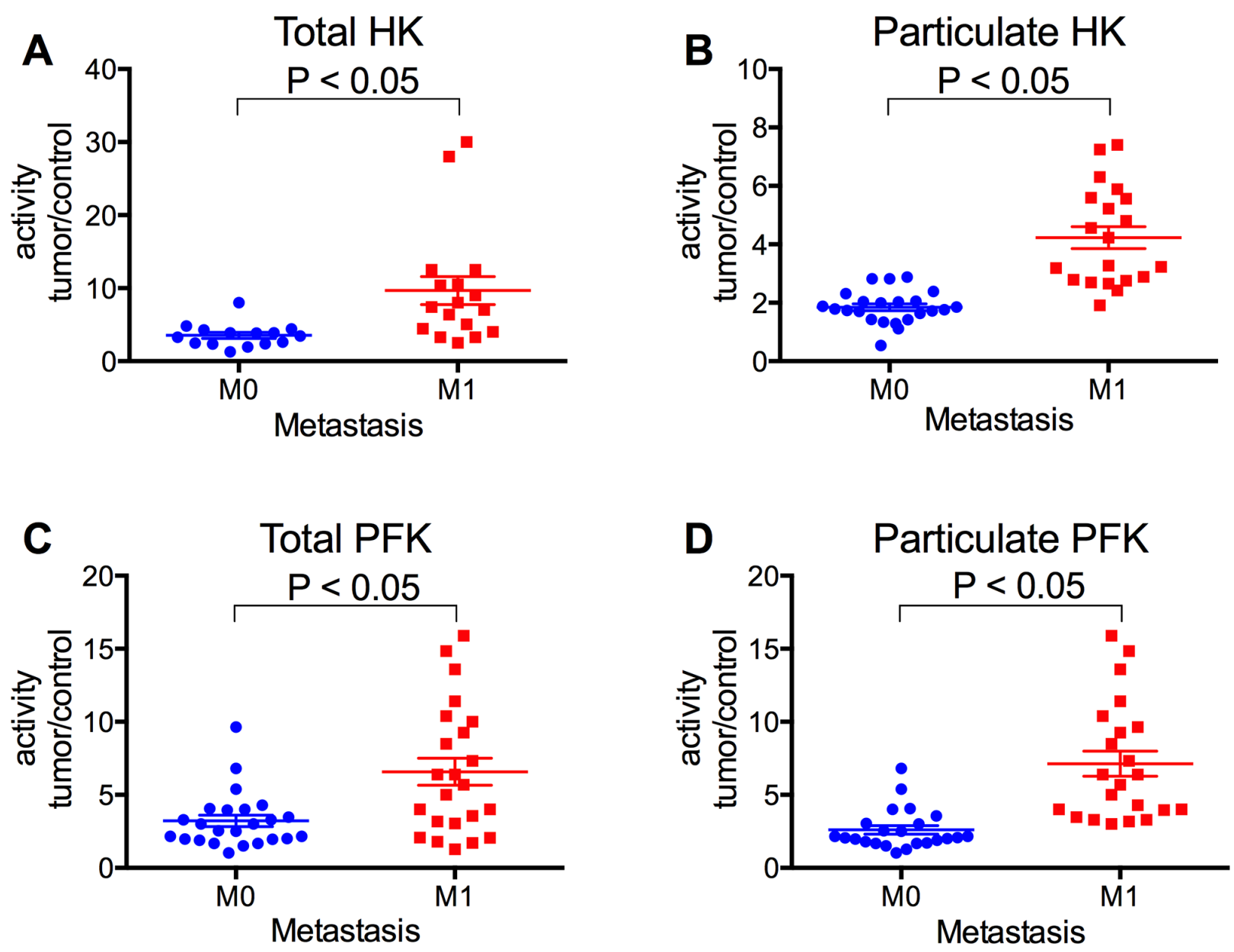

Figure 5: HK and PFK activities of the tumor samples separated with regard to the occurrence of metastasis. Samples were classified as M0 or M1 by the presence of skin metastasis in the patients as described in the Materials and Methods. HK and PFK activity in the total and particulate fractions was analyzed as described in the Materials and Methods. Panel A. Total HK activity. Panel B. Particulate HK activity. Panel C. Total PFK activity. Panel D. Particulate PFK activity. The plotted values are the mean of enzyme activity evaluated in triplicate for each tumor sample. Statistical analyses were conducted using ANOVA and Sidak's post-test.

In conclusion, the present work presents irrefutable evidence for the correlation between the activity of HK and PFK and the prognosis of breast cancer patients. Further extensive work with patients must be conducted to evaluate how the activity of these enzymes may predict the outcome. However, it is undeniable that their activity is directly correlated to the aggressiveness and invasiveness of breast cancer.

\section{MATERIALS AND METHODS}

\section{Materials}

ATP, glucose and fructose-6-phosphate (F6P) were purchased from Sigma Chemical Co. (St. Louis, MO, USA). ${ }^{32} \mathrm{Pi}$ was purchased from Instituto de Pesquisas Energéticas e Nucleares (São Paulo, Brazil). [ $\left.\gamma^{-32} \mathrm{P}\right]$ ATP was prepared according to [65]. All protein content measurements were performed as described by [66].

\section{Volunteer tissues and data collection}

All tissues were obtained from female donors along with the written consent of the patients undergoing mastectomy at Hospital Universitário Clementino Fraga Filho (HUCFF/UFRJ), Rio de Janeiro, Brazil. Tumor and control tissues from the same donors were removed during surgery. After histological analysis for the classification and grading of tumors, samples were immediately frozen in liquid $\mathrm{N}_{2}$ and stored until further use. This project was developed after the approval of the National Ethical Committee (CONEP-approval protocol 1897.0.000.197-06).

\section{Tissue fractionation}

Tissue fractionation was performed as previously described [31, 35]. After $\mathrm{N}_{2}$ withdrawal, tissues were homogenized with a Polytron homogenizer in a buffer containing $50 \mathrm{mM}$ Tris- $\mathrm{HCl}$ (pH 7.4), $0.25 \mathrm{M}$ sucrose, 

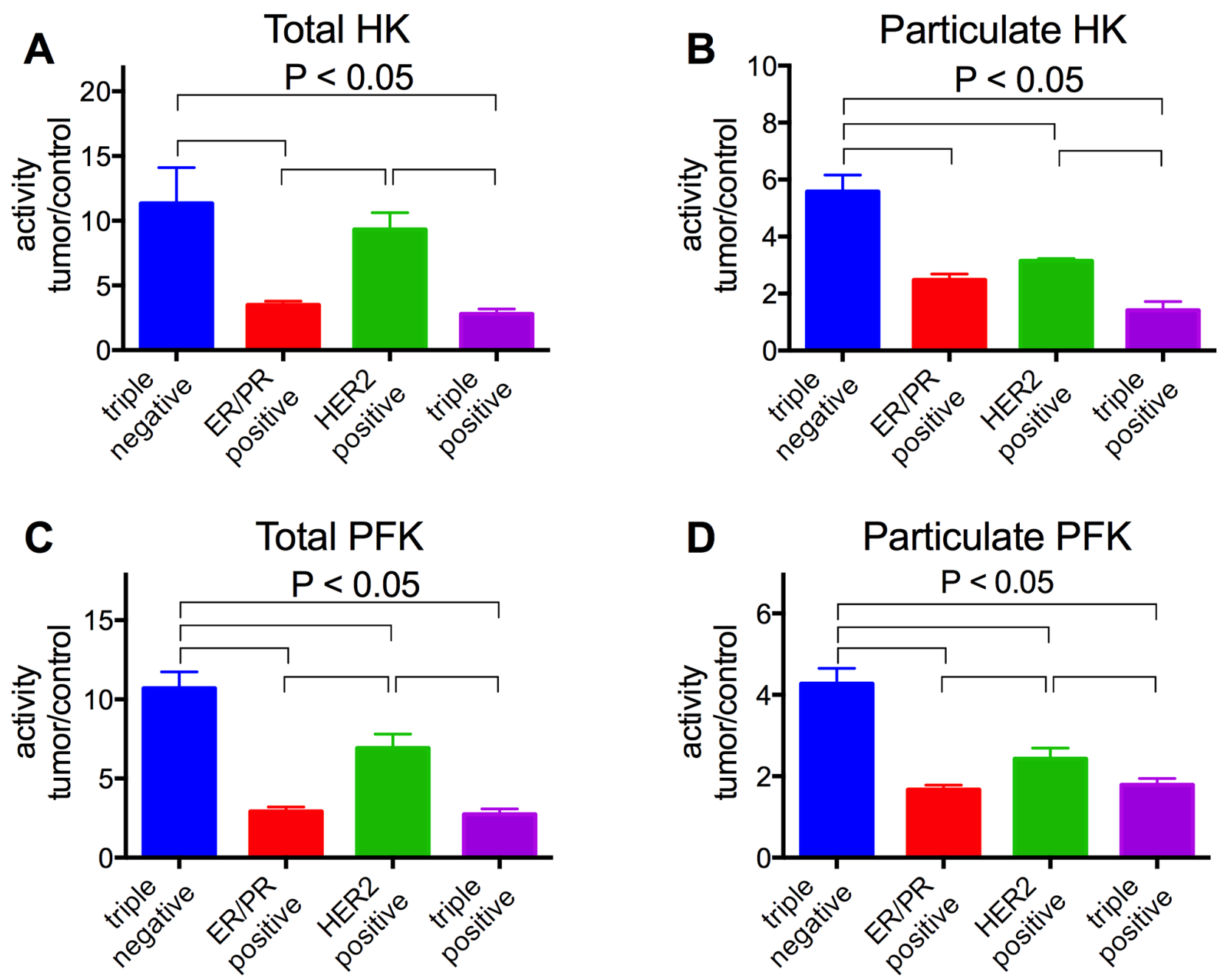

Figure 6: HK and PFK activities of the tumor samples classified according to the expression of hormone receptors. Tumor samples were stained for ER, PR and HER-2 as described in Materials and Methods. HK and PFK activities in the total and particulate fractions were analyzed as described in the Materials and Methods. Panel A. Total HK activity. Panel B. Particulate HK activity. Panel C. Total PFK activity. Panel D. Particulate PFK activity. Plotted values are the means \pm standard errors, and the numbers of cases are in accordance with the results presented in Figure 1. Statistical analyses were conducted using ANOVA and Sidak's post-test.

$20 \mathrm{mM} \mathrm{KF}, 0.2 \mathrm{mM}$ 2-mercaptoethanol and $0.5 \mathrm{mM}$ phenylmethanesulfonyl fluoride (PMSF). Homogenized tissues were centrifuged for $5 \mathrm{~min}$ at $100 \mathrm{~g}\left(4^{\circ} \mathrm{C}\right)$ for separation of cellular debris and non-digested tissues. The resultant supernatant, called total homogenate (TH), represents the total enzyme activity. The TH was centrifuged for $15 \mathrm{~min}$ at $27000 \mathrm{~g}\left(4^{\circ} \mathrm{C}\right)$, and the supernatant was centrifuged again for $45 \mathrm{~min}$ at $120.000 \mathrm{~g}\left(4^{\circ} \mathrm{C}\right)$. The resulting high-speed supernatant was used to evaluate the soluble fraction enzyme activity, while the respective pellet was resuspended in the original volume with the same buffer and used to analyze the particulate fraction enzyme activity.

\section{Immunohistochemistry}

The most representative tumor tissue block was chosen from each sample, and $5 \mu \mathrm{m}$ sections were attached to poly-L-lysine-coated slides for immunohistochemical staining. The tissue sections were deparaffinized in xylene, rehydrated in alcohol, and immersed in distilled water. The sections were then boiled for ten minutes in citrate buffer solution (10 mM, pH 6.0) in a microwave oven 3 times for epitope retrieval in staining, and the standard streptavidin-biotin immunoperoxidase method was used for immunostaining with Ki-67 antigen, HER-2, ER, PR and p53 according to [67]. The sources and dilutions of these antibodies and the epitope retrieval methods are listed in Table 1. Bound antibodies were detected using Envision TM mouse or rabbit (Dako, USA) secondary antibodies after a 45 min incubation at room temperature. Samples were considered HER-2 positive if they scored $3+$ according to the appropriate guidelines [63]. 

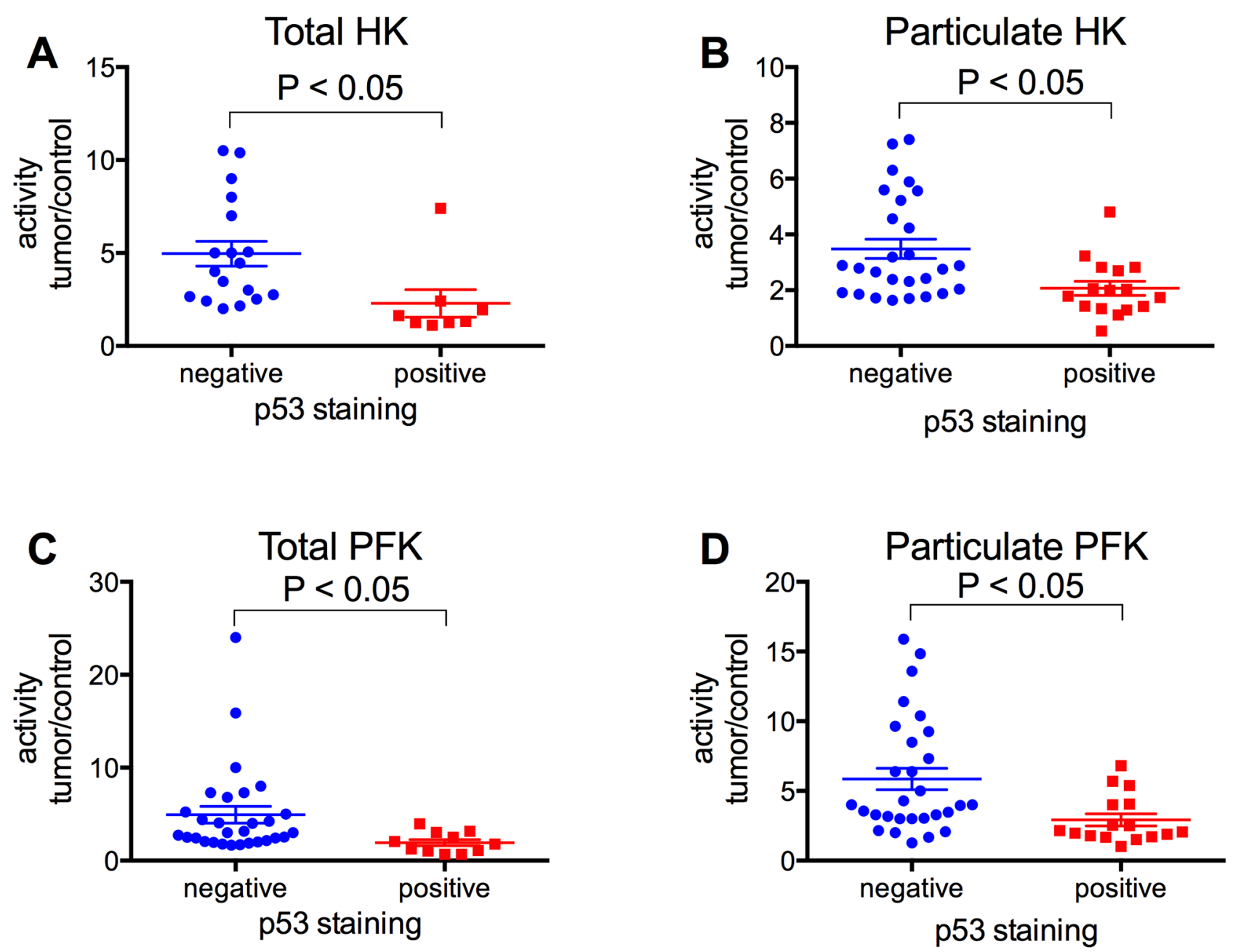

Figure 7: HK and PFK activities of the tumor samples classified according to the expression of p53. Tumor samples were stained for p53 as described in Materials and Methods. HK and PFK activity in the total and particulate fractions was analyzed as described in the Materials and Methods. Panel A. Total HK activity. Panel B. Particulate HK activity. Panel C. Total PFK activity. Panel D. Particulate PFK activity. The plotted values are the mean of enzyme activity evaluated in triplicate for each tumor sample. Statistical analyses were conducted using ANOVA and Sidak's post-test.
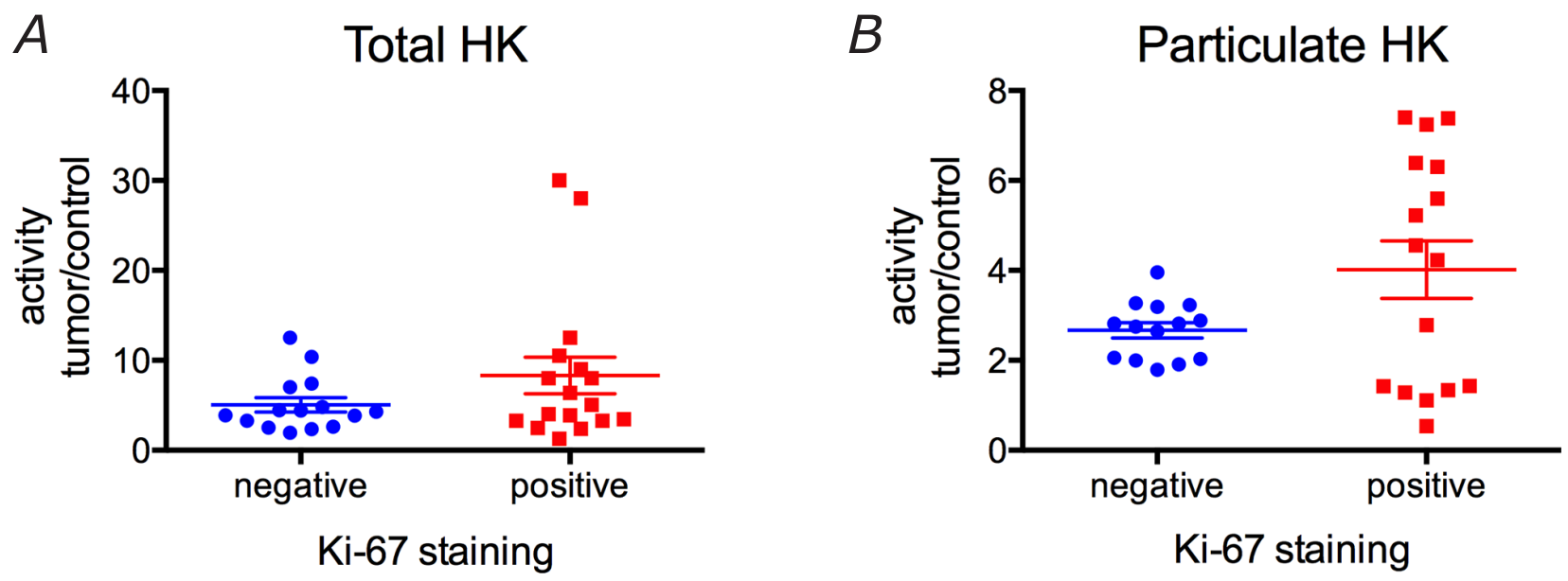

Figure 8: HK and PFK activities of the tumor samples classified according to the expression of Ki-67. Tumor samples were stained for Ki-67 as described in Materials and Methods. HK and PFK activity in the total and particulate fractions was analyzed as described in the Materials and Methods. Panel A. Total HK activity. Panel B. Particulate HK activity. (Continued) 

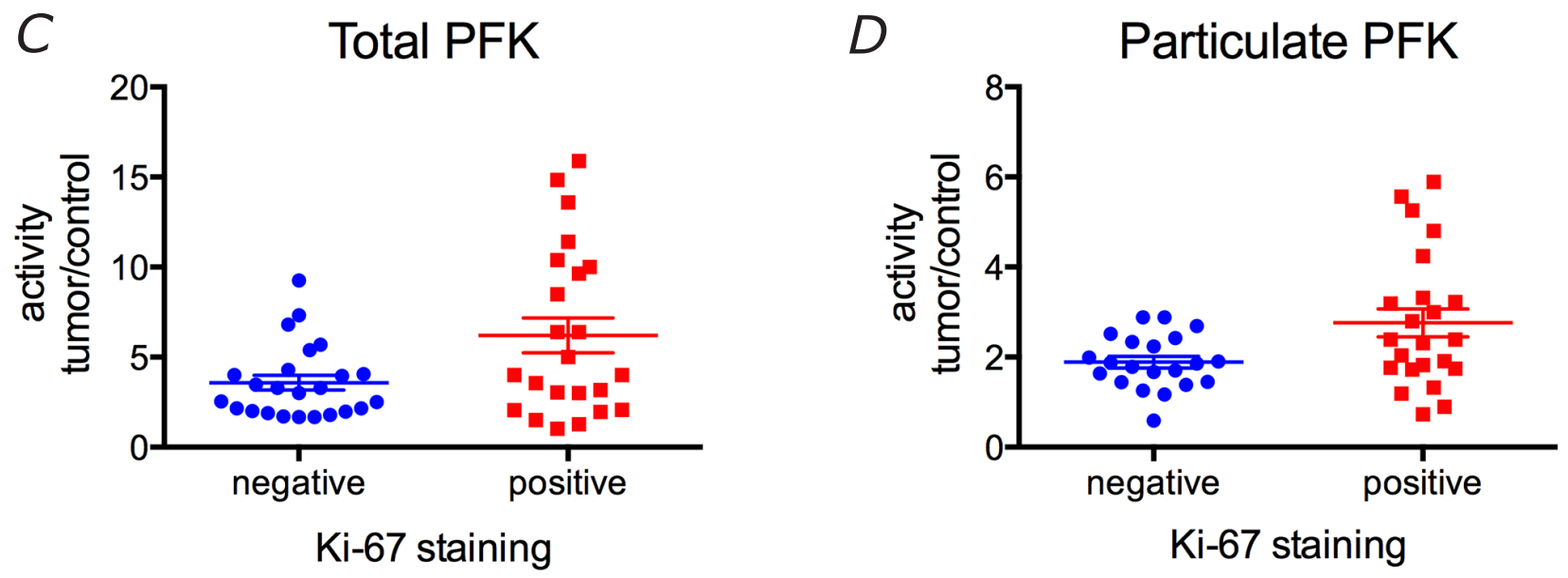

Figure 8: (Continued) HK and PFK activities of the tumor samples classified according to the expression of Ki-67. Panel C. Total PFK activity. Panel D. Particulate PFK activity. The plotted values are the mean of enzyme activity evaluated in triplicate for each tumor sample. Statistical analyses were conducted using ANOVA and Sidak's post-test.

Table 1: Details of immunohistochemical analysis

Primary antibody to
\begin{tabular}{|l|l|l|l|}
\hline Estrogen receptor (ER) & R;SP1/Thermo Scientific & 0.38889 & Pressure cooker, 9 min \\
\hline Progesterone receptor (PR) & M;PgR636/Thermo Scientific & 0.73611 & Pressure cooker, 9 min \\
\hline c-erbB2/HER-2/neu & R;SP3/Thermo Scientific & 0.11111 & Microwave oven \\
\hline p53 protein & M; DO-7/Dako & 1.91667 & Pressure cooker, 8 min \\
\hline Ki-67 antigen & M; MIB1/Dako & 0.45833 & Pressure cooker, 8 min \\
\hline
\end{tabular}

Pressure cooker: citrate buffer ( $\mathrm{pH}$ 6) (Tender Cooker, Nordic Wave, USA). Microwave: citrate buffer (pH 6), 15 min (Eletrolux, $900 \mathrm{~W}$ ).

\section{Enzymatic activity assays}

HK and PFK activities were measured by the method described in [68] with modifications introduced by [69, 70]. The reaction media contain $50 \mathrm{mM}$ Tris- $\mathrm{HCl}(\mathrm{pH} 7.4), 5$ $\mathrm{mM} \mathrm{MgCl}, 1 \mathrm{mM}\left[\gamma_{-}{ }^{32} \mathrm{P}\right]$ ATP $(4 \mu \mathrm{Ci} / \mathrm{nmol}), 50 \mu \mathrm{g} / \mathrm{ml}$ of the tissues extracts (considering their protein content), and $1 \mathrm{mM}$ fructose-6-phosphate or $5 \mathrm{mM}$ glucose, for PFK and $\mathrm{HK}$ activities, respectively. The reaction was stopped after increasing reaction times by the addition of a suspension of activated charcoal in $0.1 \mathrm{M} \mathrm{HCl}$ and $0.5 \mathrm{M}$ mannitol. After centrifugation, the supernatant products were analyzed using a liquid scintillation counter. The signals from the appropriate blanks (in the absence of fructose-6-phosphate or glucose) were measured and subtracted from all measurements to account for ATP hydrolysis. The catalytic rate was calculated by linear regression analysis of the amount of products formed vs. reaction time.

\section{Statistics}

Statistical analyses were performed using Prism 6 (GraphPad Software Inc., La Jolla, CA, USA). A two-way
ANOVA using Sidak's post-test was applied for all analyses, and the results were considered significantly different when $P<0.05$.

\section{ACKNOWLEDGMENTS}

The authors thank Dr. Carlos Bacchi (Laboratório Bacchi) for the help with the histopathology analyses.

\section{CONFLICTS OF INTEREST}

The authors declare no conflicts of interest

\section{GRANT SUPPORT}

The present work was supported by grants from Fundação Carlos Chagas Filho de Amparo a Pesquisa do Estado do Rio de Janeiro (FAPERJ), Conselho Nacional de Desenvolvimento Científico e Tecnológico (CNPq), Fundação do Câncer/Programa de Oncobiologia - UFRJ and Programa de Núcleos de Excelência (Pronex). 


\section{REFERENCES}

1. Pelicano H, Zhang W, Liu J, Hammoudi N, Dai J, Xu RH, Pusztai L, Huang P. Mitochondrial dysfunction in some triple-negative breast cancer cell lines: role of mTOR pathway and therapeutic potential. Breast cancer research. 2014; 16:434.

2. Son SH, Kim DH, Hong CM, Kim CY, Jeong SY, Lee SW, Lee J, Ahn BC. Prognostic implication of intratumoral metabolic heterogeneity in invasive ductal carcinoma of the breast. BMC cancer. 2014; 14:585.

3. Kim SK, Jung WH, Koo JS. Differential expression of enzymes associated with serine/glycine metabolism in different breast cancer subtypes. PLoS One. 2014; 9:e101004.

4. Di Leo A, Curigliano G, Dieras V, Malorni L, Sotiriou C, Swanton C, Thompson A, Tutt A, Piccart M. New approaches for improving outcomes in breast cancer in Europe. Breast. 2015; 24:321-330.

5. Balogun OD, Formenti SC. Locally advanced breast cancer strategies for developing nations. Frontiers in oncology. 2015; 5:89.

6. Tavassoli FA, Devilee P International Agency for Research on Cancer. and World Health Organization. 2003 Pathology and genetics of tumours of the breast and female genital organs. (Lyon: IARC Press).

7. Guth U, Singer G, Schotzau A, Langer I, Dieterich H, Rochlitz C, Herberich L, Holzgreve W, Wight E. Scope and significance of non-uniform classification practices in breast cancer with non-inflammatory skin involvement: a clinicopathologic study and an international survey. Annals of oncology : official journal of the European Society for Medical Oncology / ESMO. 2005; 16:1618-1623.

8. Ferreira AR, Saini KS, Metzger-Filho O. Treatment of early-stage HER2+ breast cancer-an evolving field. Ecancermedicalscience. 2015; 9:523.

9. Zardavas D, Irrthum A, Swanton C, Piccart M. Clinical management of breast cancer heterogeneity. Nature reviews Clinical oncology. 2015; 12:381-394.

10. Qiu SQ, Wei XL, Huang WH, Wu MY, Qin YS, Li YK, Zhang GJ. Diagnostic and therapeutic strategy and the most efficient prognostic factors of breast malignant fibrous histiocytoma. Scientific reports. 2013; 3:2529.

11. Mustacchi G, Biganzoli L, Pronzato P, Montemurro F, Dambrosio M, Minelli M, Molteni L, Scaltriti L. HER2positive metastatic breast cancer: A changing scenario. Critical reviews in oncology/hematology. 2015; 95:78-87.

12. Oostra DR, Macrae ER. Role of trastuzumab emtansine in the treatment of HER2-positive breast cancer. Breast cancer. 2014; 6:103-113.

13. Nazarali SA, Narod SA. Tamoxifen for women at high risk of breast cancer. Breast cancer. 2014; 6:29-36.

14. Morad SA, Cabot MC. Tamoxifen regulation of sphingolipid metabolism-therapeutic implications. Biochim Biophys Acta. 2015; 185:1134-1145.
15. Duffy MJ, Synnott NC, McGowan PM, Crown J, O’Connor D, Gallagher WM. p53 as a target for the treatment of cancer. Cancer treatment reviews. 2014; 40:1153-1160.

16. Hanahan D, Weinberg RA. Hallmarks of cancer: the next generation. Cell. 2011; 144:646-674.

17. Nikitovic D, Tzardi M, Berdiaki A, Tsatsakis A, Tzanakakis GN. Cancer microenvironment and inflammation: role of hyaluronan. Frontiers in immunology. 2015; 6:169.

18. Hsu PP, Sabatini DM. Cancer Cell Metabolism: Warburg and Beyond. Cell. 2008; 134:703-707.

19. Bayley JP, Devilee P. The Warburg effect in 2012. Current opinion in oncology. 2012; 24:62-67.

20. Kim JW, Dang CV. Cancer's molecular sweet tooth and the Warburg effect. Cancer Res. 2006; 66:8927-8930.

21. Robey IF, Stephen RM, Brown KS, Baggett BK, Gatenby RA, Gillies RJ. Regulation of the Warburg effect in early-passage breast cancer cells. Neoplasia. 2008; 10:745-756.

22. Gillies RJ, Robey I, Gatenby RA. Causes and consequences of increased glucose metabolism of cancers. J Nucl Med. 2008; 49:24S-42S.

23. Gatenby RA, Gillies RJ. Why do cancers have high aerobic glycolysis?. NatRevCancer. 2004; 4:891-899.

24. Vander Heiden MG, Cantley LC, Thompson CB. Understanding the Warburg effect: the metabolic requirements of cell proliferation. Science. 2009; 324:1029-1033.

25. Pedersen PL. Warburg, me and Hexokinase 2: Multiple discoveries of key molecular events underlying one of cancers' most common phenotypes, the "Warburg Effect", i.e., elevated glycolysis in the presence of oxygen. J Bioenerg Biomembr. 2007; 39:211-222.

26. Zancan P, Almeida FV, Faber-Barata J, Dellias JM, Sola-Penna M. Fructose-2,6-bisphosphate counteracts guanidinium chloride-, thermal-, and ATP-induced dissociation of skeletal muscle key glycolytic enzyme 6-phosphofructo-1-kinase: A structural mechanism for PFK allosteric regulation. ArchBiochemBiophys. 2007; 467:275-282.

27. Zancan P, Marinho-Carvalho MM, Faber-Barata J, Dellias JM, Sola-Penna M. ATP and fructose-2,6-bisphosphate regulate skeletal muscle 6-phosphofructo-1-kinase by altering its quaternary structure. IUBMBLife. 2008; 60:526-533.

28. Sola-Penna M, Da Silva D, Coelho WS, MarinhoCarvalho MM, Zancan P. Regulation of mammalian muscle type 6-phosphofructo-1-kinase and its implication for the control of the metabolism. IUBMB Life. 2010; 62:791-796.

29. Guzun R, Gonzalez-Granillo M, Karu-Varikmaa M, Grichine A, Usson Y, Kaambre T, Guerrero-Roesch K, Kuznetsov A, Schlattner U, Saks V. Regulation of respiration in muscle cells in vivo by VDAC through interaction with the cytoskeleton and MtCK within Mitochondrial Interactosome. Biochim Biophys Acta. 2012; 1818:1545-1554.

30. Furtado CM, Marcondes MC, Sola-Penna M, de Souza ML, Zancan P. Clotrimazole preferentially inhibits human breast 
cancer cell proliferation, viability and glycolysis. PLoS ONE. 2012; 7:e30462.

31. Coelho RG, Calaça IdC, Celestrini DdM, Correia AH, Costa MASM, Sola-Penna M. Clotrimazole disrupts glycolysis in human breast cancer without affecting non-tumoral tissues. Molecular Genetics and Metabolism. 2011; 103:394-398.

32. Zancan P, Sola-Penna M, Furtado CM, Da Silva D. Differential expression of phosphofructokinase-1 isoforms correlates with the glycolytic efficiency of breast cancer cells. Mol Genet Metab. 2010; 100:372-378.

33. Zancan P, Rosas AO, Marcondes MC, Marinho-Carvalho MM, Sola-Penna M. Clotrimazole inhibits and modulates heterologous association of the key glycolytic enzyme 6-phosphofructo-1-kinase. BiochemPharmacol. 2007; 73:1520-1527.

34. Meira DD, Marinho-Carvalho MM, Teixeira CA, Veiga VF, Da Poian AT, Holandino C, de Freitas MS, Sola-Penna M. Clotrimazole decreases human breast cancer cells viability through alterations in cytoskeleton-associated glycolytic enzymes. MolGenetMetab. 2005; 84:354-362.

35. El-Bacha T, de Freitas MS, Sola-Penna M. Cellular distribution of phosphofructokinase activity and implications to metabolic regulation in human breast cancer. MolGenetMetab. 2003; 79:294-299.

36. Penso J, Beitner R. Lithium detaches hexokinase from mitochondria and inhibits proliferation of B16 melanoma cells. MolGenetMetab. 2003; 78:74-78.

37. Penso J, Beitner R. Detachment of glycolytic enzymes from cytoskeleton of Lewis lung carcinoma and colon adenocarcinoma cells induced by clotrimazole and its correlation to cell viability and morphology. MolGenetMetab. 2002; $76: 181-188$.

38. Schwartz D, Beitner R. Detachment of the glycolytic enzymes, phosphofructokinase and aldolase, from cytoskeleton of melanoma cells, induced by local anesthetics. MolGenetMetab. 2000; 69:159-164.

39. Vertessy BG, Orosz F, Kovacs J, Ovadi J. Alternative binding of two sequential glycolytic enzymes to microtubules. Molecular studies in the phosphofructokinase/aldolase/ microtubule system. J Biol Chem. 1997; 272:25542-25546.

40. Orosz F, Vertessy BG, Salerno C, Crifo C, Capuozzo E, Ovadi J. The interaction of a new anti-tumour drug, KAR-2 with calmodulin. BrJPharmacol. 1997; 121:955-962.

41. Marin-Hernandez A, Gallardo-Perez JC, Rodriguez-Enrl̀quez S, Encalada R, Moreno-Sanchez R, Saavedra E. Modeling cancer glycolysis. Biochimica et Biophysica Acta (BBA) Bioenergetics. 2011; . DOI: 10.1016/j.bbabio.2010.11.006

42. Alves GG, Sola-Penna M. Epinephrine modulates cellular distribution of muscle phosphofructokinase. MolGenetMetab. 2003; 78:302-306.

43. Coelho WS, Costa KC, Sola-Penna M. Serotonin stimulates mouse skeletal muscle 6-phosphofructo-1-kinase through tyrosine-phosphorylation of the enzyme altering its intracellular localization. MolGenetMetab. 2007; 92:364-370.
44. Coelho WS, Da Silva D, Marinho-Carvalho MM, SolaPenna M. Serotonin modulates hepatic 6-phosphofructo1-kinase in an insulin synergistic manner. Int $\mathrm{J}$ Biochem Cell Biol. 2012; 44:150-157.

45. Coelho WS, Sola-Penna M. Serotonin regulates 6-phosphofructo-1-kinase activity in a PLC-PKC-CaMK II- and Janus kinase-dependent signaling pathway. Mol Cell Biochem. 2013; 372:211-220.

46. Da Silva D, Ausina P, Alencar EM, Coelho WS, Zancan P, Sola-Penna M. Metformin reverses hexokinase and phosphofructokinase downregulation and intracellular distribution in the heart of diabetic mice. IUBMB Life. 2012; 64:766-774.

47. Da Silva D, Zancan P, Coelho WS, Gomez LS, Sola-Penna M. Metformin reverses hexokinase and 6-phosphofructo1-kinase inhibition in skeletal muscle, liver and adipose tissues from streptozotocin-induced diabetic mouse. Arch Biochem Biophys. 2010; 496:53-60.

48. Gomez LS, Zancan P, Marcondes MC, Ramos-Santos L, Meyer-Fernandes JR, Sola-Penna M, Da Silva D. Resveratrol decreases breast cancer cell viability and glucose metabolism by inhibiting 6-phosphofructo-1-kinase. Biochimie. 2013; 95:1336-1343.

49. Grechi J, Marinho-Carvalho M, Zancan P, Cinelli LP, Gomes AMO, Rodrigues ML, Nimrichter L, Sola-Penna M. Glucuronoxylomannan from Cryptococcus neoformans Down-regulates the Enzyme 6-Phosphofructo-1-kinase of Macrophages. Journal of Biological Chemistry. 2011; 286:14820-14829.

50. Leite TC, Coelho RG, Silva DD, Coelho WS, MarinhoCarvalho MM, Sola-Penna M. Lactate downregulates the glycolytic enzymes hexokinase and phosphofructokinase in diverse tissues from mice. FEBS Letters. 2011; 585:92-98.

51. Leite TC, Da Silva D, Coelho RG, Zancan P, Sola-Penna M. Lactate favours the dissociation of skeletal muscle 6-phosphofructo-1-kinase tetramers down-regulating the enzyme and muscle glycolysis. BiochemJ. 2007; 408:123-130.

52. Marinho-Carvalho MM, Costa-Mattos PV, Spitz GA, Zancan P, Sola-Penna M. Calmodulin upregulates skeletal muscle 6-phosphofructo-1-kinase reversing the inhibitory effects of allosteric modulators. Biochimica et Biophysica Acta (BBA) - Proteins \& Proteomics. 2009; 1794:1175-1180.

53. Real-Hohn A, Zancan P, Da Silva D, Martins ER, Salgado LT, Mermelstein CS, Gomes AM, Sola-Penna M. Filamentous actin and its associated binding proteins are the stimulatory site for 6-phosphofructo-1-kinase association within the membrane of human erythrocytes. Biochimie. 2010; 92:538-544.

54. Spitz GA, Furtado CM, Sola-Penna M, Zancan P. Acetylsalicylic acid and salicylic acid decrease tumor cell viability and glucose metabolism modulating 6-phosphofructo-1-kinase structure and activity. Biochemical Pharmacology. 2009; 77:46-53.

55. Lu CL, Qin L, Liu HC, Candas D, Fan M, Li JJ. Tumor cells switch to mitochondrial oxidative phosphorylation under 
radiation via $\mathrm{mTOR}$-mediated hexokinase II inhibition-a Warburg-reversing effect. PLoS One. 2015; 10:e0121046.

56. Dai W, Wang F, Lu J, Xia Y, He L, Chen K, Li J, Li S, Liu T, Zheng Y, Wang J, Lu W, Zhou Y, Yin Q, Abudumijiti H, Chen $\mathrm{R}$, et al. By reducing hexokinase 2, resveratrol induces apoptosis in HCC cells addicted to aerobic glycolysis and inhibits tumor growth in mice. Oncotarget. 2015.

57. Xian SL, Cao W, Zhang XD, Lu YF. 3-Bromopyruvate inhibits human gastric cancer tumor growth in nude mice via the inhibition of glycolysis. Oncology letters. 2015; 9:739-744.

58. Justus CR, Sanderlin EJ, Yang LV. Molecular Connections between Cancer Cell Metabolism and the Tumor Microenvironment. Int J Mol Sci. 2015; 16:11055-11086.

59. Engel C, Fischer C. Breast cancer risks and risk prediction models. Breast care. 2015; 10:7-12.

60. Abramson VG, Lehmann BD, Ballinger TJ, Pietenpol JA. Subtyping of triple-negative breast cancer: implications for therapy. Cancer. 2015; 121:8-16.

61. Castello A, Martin M, Ruiz A, Casas AM, Baena-Canada JM, Lope V, Antolin S, Sanchez P, Ramos M, Anton A, Munoz M, Bermejo B, De Juan-Ferre A, Jara C, Chacon JI, Jimeno MA, et al. Lower Breast Cancer Risk among Women following the World Cancer Research Fund and American Institute for Cancer Research Lifestyle Recommendations: EpiGEICAM Case-Control Study. PLoS One. 2015; 10:e0126096.

62. Al-azawi D, Leong S, Wong L, Kay E, Hill AD, Young L. HER-2 positive and p53 negative breast cancers are associated with poor prognosis. Cancer investigation. 2011; 29:365-369.

63. Wolff AC, Hammond ME, Schwartz JN, Hagerty KL, Allred DC, Cote RJ, Dowsett M, Fitzgibbons PL, Hanna WM, Langer A, McShane LM, Paik S, Pegram MD, Perez EA, Press MF, Rhodes A, et al. American Society of Clinical Oncology/College of American Pathologists guideline recommendations for human epidermal growth factor receptor 2 testing in breast cancer. Archives of pathology \& laboratory medicine. 2007; 131:18-43.

64. Kim T, Han W, Kim MK, Lee JW, Kim J, Ahn SK, Lee HB, Moon HG, Lee KH, Kim TY, Han SW, Im SA, Park IA, Kim JY, Noh DY. Predictive Significance of p53, Ki-67, and Bcl-2 Expression for Pathologic Complete Response after Neoadjuvant Chemotherapy for Triple-Negative Breast Cancer. Journal of breast cancer. 2015; 18:16-21.

65. Maia JCC, Gomes SL, Juliani MH, Morel CM. Preparation of and [a- $32 \mathrm{P}$ ]-nucleoside triphosphate, with high specific activity. Genes and Antigenes of Parasites: a Laboratory Manual. Rio de Janeiro, Brazil: FIOCRUZ1983; 146-157.

66. Lowry OH, Rosebrough NJ, Farr AL, Randall RJ. Protein measurement with the Folin phenol reagent. Journal of Biological Chemistry. 1951; 193:265-275.

67. Bacchi LM, Corpa M, Santos PP, Bacchi CE, Carvalho FM. Estrogen receptor-positive breast carcinomas in younger women are different from those of older women: a pathological and immunohistochemical study. Breast. 2010; 19:137-141.

68. Sola-Penna M, dos Santos AC, Alves GG, El-Bacha T, Faber-Barata J, Pereira MF, Serejo FC, Da Poian AT, Sorenson M. A radioassay for phosphofructokinase-1 activity in cell extracts and purified enzyme. JBiochemBiophysMethods. 2002; 50:129-140.

69. Zancan P, Sola-Penna M. Calcium influx: a possible role for insulin modulation of intracellular distribution and activity of 6-phosphofructo-1-kinase in human erythrocytes. MolGenetMetab. 2005; 86:392-400.

70. Zancan P, Sola-Penna M. Regulation of human erythrocyte metabolism by insulin: cellular distribution of 6-phosphofructo-1-kinase and its implication for red blood cell function. MolGenetMetab. 2005; 86:401-411. 